\title{
Spatially-discretized high-temperature approximations and their $O(N)$ implementation on a grid
}

\author{
Cristian Predescu* \\ Department of Chemistry and Kenneth S. Pitzer Center for Theoretical Chemistry, \\ University of California, Berkeley, California 94720
}

(Dated: December 13, 2006)

\begin{abstract}
We consider the problem of performing imaginary-time propagation of wavefunctions on a grid. We demonstrate that spatially-continuous high-temperature approximations can be discretized in such a way that their convergence order is preserved. Requirements of minimal computational work and reutilization of data then uniquely determine the optimal grid, quadrature technique, and propagation method. It is shown that the optimal propagation technique is $O(N)$ with respect to the grid size. The grid technique is utilized to compare the Monte Carlo efficiency of the Trotter-Suzuki approximation against a recently introduced fourth-order high-temperature approximation, while circumventing the issue of statistical noise, which usually prevents such comparisons from being carried out. We document the appearance of a systematic bias in the Monte Carlo estimators that involve temperature differentiation of the density matrix, bias that is due to the dependence of the eigenvalues on the inverse temperature. This bias is negotiated more successfully by the short-time approximations having higher convergence order, leading to non-trivial computational savings.
\end{abstract}

PACS numbers: 05.30.-d, 02.70.Ss

\section{INTRODUCTION}

Although the evaluation of imaginary-time path integrals is normally performed by Monte Carlo techniques, ${ }^{1}$ there is also substantial interest in the numerical implementation on a grid. The strongest motivation is provided by the fact that real-time correlation functions are usually computed for physical systems at finite temperature. Even when this is not the case, imaginarytime propagation can be utilized to filter out the contribution of the high-energy eigenfunctions, whether for the purpose of diagonalizing the Hamiltonian or evaluating real-time correlation functions. This contribution appears whenever the initial function is not sufficiently smooth. In this respect, it is worth mentioning that substantial contributions of the high-energy eigenfunctions may be artificially retained if the propagation in imaginary-time is not performed accurately. Several other research problems may benefit from efficient imaginary-time propagation on an optimally chosen grid. For example, grid methods can be utilized to produce highly accurate thermodynamic data for small molecules or to solve temperature-dependent Hartree equations. ${ }^{2}$

Given its widespread use together with the Monte Carlo integration technique, it is a little bit surprising that efficient propagation in imaginary time is rather costly to perform on a grid. Historically, this difficulty has been caused by the lack of high-order stable shorttime approximations for the imaginary-time propagator (density matrix). For real-time propagation, the appropriate stability condition is the unitarity of the shorttime approximation. The existence of symplectic integral schemes of arbitrary order is by now an well-established result. $^{3-5}$ Thus, there are real numbers $a_{0}, a_{1}, \ldots, a_{l}$ and $b_{1}, b_{2}, \ldots, b_{l}$ such that

$$
\begin{aligned}
e^{-\beta(K+V)=} & e^{-a_{0} \beta V} e^{-b_{1} \beta K} e^{-a_{1} \beta V} \ldots \\
& \ldots e^{-b_{l} \beta K} e^{-a_{l} \beta V}\left[1+O\left(\beta^{\nu+1}\right)\right],
\end{aligned}
$$

for any convergence order $\nu$. Because $\beta=i t / \hbar$, the righthand side of Eq. (1) is a composition of unitary operators, consequently, a unitary operator by itself. The numerical implementation on a grid of symplectic integrators can be performed by fast Fourier transform (FFT) in such a way that unitarity is preserved. ${ }^{6-8}$ The count of operations for a propagation step is $O\left(N \log _{2}(N)\right)$ with respect to the grid size $N$. As is the case with pseudospectral methods, the convergence is faster than any polynomial with respect to $N$ (assuming periodic boundary conditions). ${ }^{9}$ With respect to the Trotter index $n$, the convergence is only polynomial, being given by $O\left(1 / n^{\nu}\right)$.

For imaginary-time propagation, the stability conditions are given by the requirement that the numbers $a_{0}, a_{1}, \ldots, a_{l}$ and $b_{1}, b_{2}, \ldots, b_{l}$ be also positive. Unfortunately, Suzuki ${ }^{5}$ has shown that no such numbers exist for orders of convergence greater or equal to 3. More recently, Goldman and Kaper ${ }^{10}$ have demonstrated that at least a pair $\left(a_{i}, b_{j}\right)$ must be negative. Because the kinetic operator is unbounded, no stable decompositions of order greater or equal to 3 exist even for potentials that are bounded both from below and above. These results have meant that the use of symplectic integral schemes has either been limited to the trapezoidal splitting

$$
e^{-\beta(K+V)}=e^{-\frac{1}{2} \beta V} e^{-\beta K} e^{-\frac{1}{2} \beta V}\left[1+O\left(\beta^{3}\right)\right]
$$

or discarded altogether in favor of techniques that treat the imaginary and real time propagation on a more or less equal footing. Surveys of the techniques available in the chemical physics literature can be found in Kosloff's review $^{8}$ or Mazziotti's comment. ${ }^{11}$ We shall refer to the 
short-time approximation described by Eq. (2) as the Trotter-Suzuki approximation. At this point however, we break away from the field of quantum dynamical methods and restrict our attention to imaginary-time propagation alone. We use Suzuki's negative result to substantiate the view that an efficient algorithm must treat the real and imaginary time propagation differently, since results from one world do not necessarily have a correspondent in the other.

The concepts of "unitarity" and "positivity" will be utilized many times because, roughly, they also describe those operations that are numerically stable when performed on a computer: multiplication of unitary matrices and addition of positive numbers. As such, the real-time propagation is best performed through unitary operations. Since this requirement may sometimes conflict with the efficiency of a numerical scheme, one is allowed to stray away a little bit and recover unitarity asymptotically only. Likewise, the implementation of the imaginary-time propagation solely by addition of positive numbers, although desirable for reasons of numerical stability, may be relaxed a little bit for the sake of numerical efficiency. Nevertheless, in the present development, we shall respect the requirement that the imaginary-time propagation must be performed by addition of positive numbers only and try to see how far we can get under this constraint.

We shall demonstrate that the imaginary-time propagation can be performed in a number of operations that scales linearly with the number of grid points, while preserving the convergence order as well as the positivity of the short-time approximation employed. We shall also demonstrate that there are implementations that make the density matrix sparse, with a number of interaction sites scaling according to the law $k^{d}$, where $k$ is a small number depending on the order of convergence (for example, 3 for order of convergence two and 7 for order of convergence four) and $d$ is the dimensionality of the system. The optimal grid is an equidistant one, with the spacing between successive knots related by strict formulas to the inverse temperature. The memory requirement is $3^{d} N$ for second order and $7^{d} N$ for fourth order shorttime approximations, where $N=N_{1} N_{2} \cdots N_{d}$ is the grid size. The computational effort for a single propagation step is given by the same values. We also argue that more efficient propagation schemes might be found in the future, schemes for which the sparsity of the density matrix grows only polynomially with the dimensionality of the system. As a local method, the numerical technique developed here can easily handle complex geometry and boundary constraints.

The correctness of the theory is verified numerically for one, two, and three dimensional examples. We utilize the numerical accuracy of the grid techniques to quantify the Monte Carlo efficiency of a recently introduced fourth-order short-time approximation ${ }^{21}$ versus the Trotter-Suzuki approximation. Such studies are difficult to undertake during the actual Monte Carlo simula- tion because the statistical noise prevents us from obtaining data accurate enough to verify the convergence order in a way that cannot be questioned. The comparison is facilitated by the fact that the computational effort for the fourth-order technique is exactly three times larger than that for the Trotter-Suzuki approximation, again, as far as Monte Carlo simulations are concerned. We demonstrate that the Monte Carlo estimators obtained by temperature differentiation of the density matrix or partition function exhibit a systematic bias that does not appear in their spectral counterparts, a bias which represents the main source of errors. As the Trotter index $n$ is increased, this bias vanishes as fast as $O\left(1 / n^{\nu}\right)$, where $\nu$ is the convergence order of the short-time approximation. Because of this dependence, the fourth-order short-time approximation is more successful in coping with the systematic errors, an assertion that is fully supported by the numerical results.

\section{SPATIALLY-DISCRETIZED SHORT-TIME APPROXIMATIONS OF PRESCRIBED CONVERGENCE ORDER}

We commence this section by reviewing some of the basic results concerning the evaluation of wavefunctions by propagation in imaginary-time via a Lie-Trotter product. Then, we generalize the Lie-Trotter product in a way that is conducive of actual numerical implementations on a grid. The generalization can also be understood as a technique of altering a short-time approximation with preservation of both the positivity and the convergence order of the original spatially-continuous approximations.

Let $\rho\left(x, x^{\prime} ; \beta\right)$ denote the density matrix of a onedimensional quantum mechanical system, that is, the time-dependent Green's function of the Bloch equation

$$
-\frac{\hbar^{2}}{2 m_{0}} \frac{\partial^{2}}{\partial x^{2}} \psi_{\beta}(x)+V(x) \psi_{\beta}(x)=-\frac{\partial}{\partial \beta} \psi_{\beta}(x),
$$

with initial value $\psi_{\beta}(x)=\psi_{0}(x)$ and appropriate Dirichelet boundary conditions. If the density matrix is known, then the identity

$$
\psi_{\beta}(x)=\int_{\mathbb{R}} \rho\left(x, x^{\prime} ; \beta\right) \psi_{0}\left(x^{\prime}\right) d x^{\prime}
$$

provides a means of recovering the value of the imaginary-time propagated wavefunction $\psi_{\beta}(x)$ by computing a one-dimensional integral. We recall the semigroup property of the density matrix:

$$
\rho\left(x, x^{\prime} ; \beta_{1}+\beta_{2}\right)=\int_{\mathbb{R}} \rho\left(x, x^{\prime \prime} ; \beta_{1}\right) \rho\left(x^{\prime \prime}, x^{\prime} ; \beta_{2}\right) d x^{\prime \prime},
$$

for all $\beta_{1}, \beta_{2} \geq 0$. If applied iteratively, the semigroup property leads to the following algorithm for the evaluation of time-dependent wavefunctions. Starting with $\psi_{0}(x)$, iterate the transformation

$$
\left\{\begin{array}{l}
\psi_{\tau}(x)=\int_{\mathbb{R}} \rho\left(x, x^{\prime} ; \tau\right) \psi_{0}\left(x^{\prime}\right) d x^{\prime} \\
\psi_{0}(x)=\psi_{\tau}(x)
\end{array}\right.
$$


a number of $n$ times, with $\tau$ set to the value $\tau_{n}=\beta / n$. The quantity $\tau_{n}$ represents a time step that can be made arbitrarily small by increasing $n$.

The Trotter theorem ${ }^{12}$ provides a means of generalizing the algorithm expressed by Eq. (6) so that to also encompass a certain class of short-time approximations $\rho_{0}\left(x, x^{\prime} ; \tau\right)$ to the density matrix. Thus, if $\psi_{\beta}^{(n)}(x)$ represents the $n$-fold iteration of the algorithm

$$
\left\{\begin{array}{l}
\psi_{\tau}^{\prime}(x)=\int_{\mathbb{R}} \rho_{0}\left(x, x^{\prime} ; \tau\right) \psi_{0}\left(x^{\prime}\right) d x^{\prime} \\
\psi_{0}(x)=\psi_{\tau}^{\prime}(x)
\end{array}\right.
$$

for $\tau=\tau_{n}$, then the Trotter theorem asserts that $\psi_{\beta}^{(n)}(x) \rightarrow \psi_{\beta}(x)$ as $n \rightarrow \infty$ in the sense of $L^{2}(\mathbb{R})$ convergence. The Trotter theorem applies provided that $\rho_{0}\left(x, x^{\prime} ; \tau\right)$ is of the form given by Eq. (1) and the potential $V(x)$ is continuous and bounded from below. Nevertheless, more general constructions of short-time approximations are available. The pointwise and norm convergence of the resulting Lie-Trotter products can be justified by means of the Feynman-Kac formula. A short-time approximation $\rho_{0}\left(x, x^{\prime} ; \tau\right)$ is said to have convergence order $\nu$ if

$$
\psi_{\beta}^{(n)}(x)=\psi_{\beta}(x)+O\left(1 / n^{\nu}\right)
$$

for all square integrable initial functions $\psi_{0}(x)$ and positive inverse temperatures $\beta$.

Eq. (7) can be cast in a form which, as we shall see, is more amenable to grid implementations. Because the density matrix of a free particle

$$
\rho_{f p}\left(x, x^{\prime} ; \tau\right)=\left(2 \pi \sigma^{2}\right)^{-1 / 2} \exp \left[-\left(x^{\prime}-x\right)^{2} /\left(2 \sigma^{2}\right)\right],
$$

with $\sigma=\left(\hbar^{2} \tau / m_{0}\right)^{1 / 2}$, is strictly positive, we can write

$$
\rho_{0}\left(x, x^{\prime} ; \tau\right)=\rho_{f p}\left(x, x^{\prime} ; \tau\right) r_{0}\left(x, x^{\prime} ; \tau\right)
$$

as well as

$$
\rho\left(x, x^{\prime} ; \tau\right)=\rho_{f p}\left(x, x^{\prime} ; \tau\right) r\left(x, x^{\prime} ; \tau\right) .
$$

An initial function $\psi_{0}(x)$ is evolved by the exact propagator according to the equation

$$
\begin{aligned}
& \psi_{\tau}(x)=\int_{\mathbb{R}} \rho\left(x, x^{\prime} ; \tau\right) \psi_{0}\left(x^{\prime}\right) d x^{\prime} \\
& =\int_{\mathbb{R}} \frac{1}{\sqrt{2 \pi \sigma^{2}}} e^{-\left(x^{\prime}-x\right)^{2} /\left(2 \sigma^{2}\right)} r\left(x, x^{\prime} ; \tau\right) \psi_{0}\left(x^{\prime}\right) d x^{\prime} \\
& =\int_{\mathbb{R}} \frac{1}{\sqrt{2 \pi}} e^{-z^{2} / 2} r(x, x+z \sigma ; \tau) \psi_{0}(x+z \sigma) d z,
\end{aligned}
$$

in the infinitesimal future $\tau$. Using the notation

$$
d \mu(z)=(2 \pi)^{-1 / 2} \exp \left(-z^{2} / 2\right) d z
$$

for the respective Gaussian measure, we can put Eq. (6) in the form

$$
\psi_{\tau}(x)=\int_{\mathbb{R}} r(x, x+z \sigma ; \tau) \psi_{0}(x+z \sigma) d \mu(z) .
$$

By a similar reasoning, the short-time approximation $\rho_{0}\left(x, x^{\prime} ; \tau\right)$ evolves the initial function according to the law

$$
\psi_{\tau}^{\prime}(x)=\int_{\mathbb{R}} r_{0}(x, x+z \sigma ; \tau) \psi_{0}(x+z \sigma) d \mu(z) .
$$

Having reached this point in our presentation, we introduce a generalization of the Lie-Trotter product as the $n$-fold iteration of the algorithm

$$
\left\{\begin{array}{l}
\psi_{\tau}^{\prime \prime}(x)=\int_{\mathbb{R}} r_{0}(x, x+z \sigma ; \tau) \psi_{0}(x+z \sigma) d \mu_{0}(z), \\
\psi_{0}(x)=\psi_{\tau}^{\prime \prime}(x),
\end{array}\right.
$$

again with $\tau=\tau_{n}=\beta / n$. The quantity $d \mu_{0}(z)$ is some positive distribution that approximates the Gaussian measure defined by Eq. (13). The following theorem provides the necessary and sufficient conditions that the measure $d \mu_{0}(z)$ must satisfy in order for the resulting product to converge to the exact wavefunction as fast as $O\left(1 / n^{\nu}\right)$.

Theorem 1 Assume that the positive (non-negative) measure $d \mu_{0}(z)$ integrates exactly all polynomials of order less or equal to $2 \nu+1$. That is,

$$
\int_{\mathbb{R}} z^{k} d \mu_{0}(z)=\frac{1}{\sqrt{2 \pi}} \int_{\mathbb{R}} z^{k} e^{-z^{2} / 2} d z, \quad \forall 0 \leq k \leq 2 \nu+1 .
$$

Assume also that the convergence order of the shorttime approximation $\rho_{0}\left(x, x^{\prime} ; \beta\right)$ is $\nu$. Then the short-time propagation defined by

$$
\psi_{\tau}^{\prime \prime}(x)=\int_{\mathbb{R}} r_{0}(x, x+z \sigma ; \tau) \psi_{0}(x+z \sigma) d \mu_{0}(z)
$$

preserves the order of convergence $\nu$.

In order not to interrupt the flow of the presentation, we perform the proof of the theorem in Appendix I. By preservation of the convergence order we understand that the identity expressed by Eq. (8) remains true provided that $\psi_{\beta}^{(n)}(x)$ is now regarded as the $n$-fold iteration of the algorithm defined by Eq. (16). Theorem 1 shows that any short-time approximation of order $\nu$ can be spatially discretized in such a way that the imaginary-time propagation defined by Eq. (18) preserves both the positivity and the convergence order. A well-known example of discrete distributions $d \mu_{0}(z)$ satisfying Eq. (17) is represented by the Gauss-Hermite quadrature scheme specified by a number of $\nu+1$ positive weights $w_{i}^{h}$ and knots $z_{i}^{h}$. In terms of Dirac's delta functions, the distribution $d \mu_{0}(z)$ reads

$$
d \mu_{0}(z)=\sum_{j=1}^{\nu+1} w_{j}^{h} \delta\left(z-z_{j}^{h}\right) d z .
$$

Therefore, the time-propagation defined by Eq. (18) becomes

$$
\psi_{\tau}^{\prime \prime}(x)=\sum_{j=1}^{\nu+1} w_{j}^{h} r_{0}\left(x, x+z_{j}^{h} \sigma ; \tau\right) \psi_{0}\left(x+z_{j}^{h} \sigma\right) .
$$


For $d$-dimensional systems, Theorem 1 remains true in the sense that the measure $d \mu_{0}(z)$ should be chosen such that it integrates exactly all $d$-dimensional polynomials of degree less or equal to $2 \nu+1$. The most simple cubature scheme satisfying these requirements is obtained by considering a one-dimensional Gauss-Hermite quadrature scheme for each dimension. The number of terms in the sum appearing in Eq. $(20)$ becomes $(\nu+1)^{d}$. However, although this is the strategy employed in the present work, the cubature technique presented is not the optimal Gauss-Hermite cubature scheme. It has been long conjectured that the optimal Gauss-Hermite cubature scheme only needs a number of terms that scales polynomially as $d^{\nu}$. If the requirements that the weights be all positive is relaxed, then a plethora of cubature schemes scaling as $d^{\nu}$ are known. The ones documented by Cools ${ }^{13-15}$ have a high quality, as measured by the percentage of negative sign in their weights. In fact, for small $d$, many of them exhibit positive weights only. In a seminal paper, ${ }^{16}$ Victoir explictly constructs a cubature scheme of order 5 having $d^{2}$ scaling. Still, whether or not the polynomial degree is generally $\nu$ remains an open question. Regarding the present work, a problem that is still to be addressed is whether or not there are cubature techniques that are centrally invariant and such that the cubature knots can be arranged in a $d$-dimensional regular pattern that covers the domain delimited by the boundary conditions. A centrally invariant cubature technique ensures that the linear transformation defined by Eq. (18) is Hermitian. The reader will understand the relevance of a regular pattern from the requirement of reutilization of data, which is utilized in the following section. In the same reference, Victoir constructs a polynomially scaling cubature scheme of order 5 satisfying all these constraints. The scheme has $d^{3}$ scaling, is nearly optimal for $d \leq 7$, and will be investigated in future work.

\section{GRID IMPLEMENTATION OF THE TROTTER-SUZUKI APPROXIMATION}

The grid technique demonstrated in the present section applies to all short-time approximations of convergence order $\nu=2$. Nevertheless, due to its widespread use, the Trotter-Suzuki approximation will constitute the only example utilized. For this approximation, we have

$$
r_{0}\left(x, x^{\prime} ; \tau\right)=e^{-\tau\left[V(x)+V\left(x^{\prime}\right)\right] / 2} .
$$

According to the mathematical derivation presented in the preceding section, the most economical propagation scheme that preserves the convergence order is the 3point Gauss-Hermite quadrature technique. Therefore, we have

$$
\psi_{\tau}^{\prime \prime}(x)=\sum_{j=-1}^{1} w_{j}^{h} r_{0}\left(x, x+j h_{0} \sigma ; \tau\right) \psi_{0}\left(x+j h_{0} \sigma\right)
$$

where $w_{-1}^{h}=w_{1}^{h}=1 / 6, w_{0}^{h}=2 / 3$, and $h_{0}=$ $\sqrt{3}$. Eq. (22) implicitly defines the spatial discretization of the short-time approximation, through the values $w_{-1}^{h} r_{0}\left(x, x-h_{0} \sigma ; \tau\right), w_{0}^{h} r_{0}(x, x ; \tau)$, and $w_{1}^{h} r_{0}(x, x+$ $\left.h_{0} \sigma ; \tau\right)$. Due to the central invariance of the GaussHermite quadrature scheme, the linear transformation specified by Eq. (22) is Hermitian.

In order to compute the value of the wavefunction at the point $x$ and at the time $\tau$, we only need to know the values of the initial wavefunction at the three points $x-$ $h_{0} \sigma, x$, and $x+h_{0} \sigma$. One quickly realizes that, for reasons of efficiency, the grid must be chosen to be equidistant, with the mesh given by the equation

$$
\Delta=\sqrt{3} \sigma=\left(3 \hbar^{2} \tau / m_{0}\right)^{1 / 2}
$$

Indeed, let us assume that we have advanced $k$ shorttime propagation steps and we are now at the step $k+1$. In order to evaluate the function at the grid point $x$, we need the values of the previous function at the grid points $x-h_{0} \sigma, x$, and $x+h_{0} \sigma$. If these values are not on the grid (and, therefore, were not computed at the iteration step $k$ ), we must go back in time and compute them anew. Clearly, such an approach is computationally inefficient and only the points on the grid are to be utilized. Therefore, $h_{0} \sigma$ must be a multiple of the mesh size $\Delta$. However, again for reasons of computational efficiency, we are free to choose the largest mesh compatible with this constraint. Hence, the value $\Delta=\sqrt{3} \sigma$ is obtained.

The propagation technique defined by Eq. (22) can also be conceived as a matrix multiplication ${ }^{17}$ with a sparse matrix (band-diagonal for one-dimensional systems). Several authors ${ }^{18-20}$ have also noticed that the imaginary-time propagation becomes unstable if the grid mesh is not taken to be of the form

$$
\Delta=\epsilon_{0}\left(\hbar^{2} \tau / m_{0}\right)^{1 / 2} \text {. }
$$

In the context of the trapezoidal quadrature technique, the various authors have proposed different values for the parameter $\epsilon_{0}$ in order to maintain high accuracy. As such, Thirumalai et $a l^{18}$ propose a value of $\epsilon_{0}=$ $(2 \cdot 0.145)^{1 / 2}=0.539$, whereas Sethia et $a l^{19}$ argue for $\epsilon_{0}=(2 \cdot 0.25)^{1 / 2}=0.707$. By comparison, the present technique allows for a larger mesh size corresponding to $\epsilon_{0}=3^{1 / 2}=1.732$, while not only preserving the convergence order, but also ensuring a sparse representation of the density matrix. Clearly, due to Theorem 1 and the optimality of the Gauss-Hermite quadrature technique, no larger mesh that preserves the convergence order is possible.

For multidimensional systems, the grid mesh varies according to the mass of the particles associated with each degree of freedom:

$$
\Delta_{k}=\sqrt{3} \sigma_{k}=\left(3 \hbar^{2} \tau / m_{0, k}\right)^{1 / 2}, \quad 1 \leq k \leq d
$$

The propagation is obtained by summing over all $3^{d}$ 
neighboring sites. For a two-dimensional system, we have

$$
\begin{array}{r}
\psi_{\tau}^{\prime \prime}(x, y)=\sum_{j_{1}, j_{2}=-1}^{1} w_{j_{1}}^{h} w_{j_{2}}^{h} r_{0}\left(x, y, x+j_{1} \Delta_{1}, y+j_{2} \Delta_{2} ; \tau\right) \\
\times \psi_{0}\left(x+j_{1} \Delta_{1}, y+j_{2} \Delta_{2}\right) .
\end{array}
$$

If the grid has a total of $N=N_{1} N_{2} \ldots N_{d}$ points, then a propagation step takes a number proportional to $3^{d} N=\left(3 N_{1}\right) \cdot\left(3 N_{2}\right) \cdots\left(3 N_{d}\right)$ multiplications and additions. This scaling is more favorable than the $N \log _{2}(N)$ scaling obtained by fast Fourier transform (not counting the overhead). More importantly, the propagation scheme is amenable to any type of boundary conditions. In particular, Dirichelet boundary conditions can easily be implemented by setting the value $r_{0}\left(x, y, x^{\prime}, y^{\prime} ; \tau\right)$ equal to zero whenever $(x, y)$ or $\left(x^{\prime}, y^{\prime}\right)$ exceed the boundary.

With these clarifications for high-dimensional systems, from now on, we shall utilize a one-dimensional notation only. For definiteness, we let $x_{1}, \ldots, x_{N}$ be the grid points. An object one is often interested in computing is the density matrix $\rho\left(x, x^{\prime} ; \beta\right)$ on the grid. Regarded as a function of $x$, the density matrix represents the wavefunction obtained by propagating a delta function localized at $x^{\prime}$. On a grid, the delta function can be approximated by a square of base $\Delta$ and height $1 / \Delta$ (since it must integrate to 1 ) centered about $x^{\prime}$. Thus, the values of the density matrix on the grid, that is the quantities $\rho\left(x_{i}, x_{j} ; \beta\right)$ for $i, j=1,2, \ldots, N$, can be obtained by propagating functions that have value $1 / \Delta$ at the position $j$ and zero elsewhere. From the density matrix, one may evaluate other quantities of interest, such as the partition function

$$
Z_{n}(\beta)=\Delta \sum_{i=1}^{N} \rho_{n}\left(x_{i}, x_{i} ; \beta\right)
$$

The approximation to the partition function designated by $n$ is obtained by time-propagating a short-time approximation of inverse temperature $\tau_{n}=\beta / n$ exactly $n$ times. Notice that, according to Eq. (23), the mesh of the grid decreases as the square root of the number of time slices. In turn, for a one-dimensional system residing between the end points $a$ and $b$, the number of grid points utilized increases as the square root of the number of time slices, according to the formula

$$
N \approx \frac{b-a}{\Delta}=\left[\frac{(b-a)^{2} m_{0} n}{3 \hbar^{2} \beta}\right]^{1 / 2} .
$$

\section{GRID IMPLEMENTATION OF FOURTH-ORDER SHORT-TIME APPROXIMATIONS}

Fourth-order short-time approximations require the use of minimalist discrete measures $d \mu_{0}(z)$ that exactly integrate all polynomials up to order 9. An example is provided by the Gauss-Hermite quadrature in 5 points. Unfortunately, the quadrature knots are not evenly distributed, in the sense that they are not a multiple of a common mesh. As such, we start the present section by designing a 7-point Hermite quadrature technique that has equally spaced knots. Because the quadrature is taken to be centrally invariant (so that $w_{j}^{h}=w_{-j}^{h}$ and $z_{j}^{h}=j h_{0}=-z_{-j}^{h}$, for $\left.j=0,1,2,3\right)$, all the odd-order monomials are exactly integrated (to zero). The remaining monomials lead to the system of equations

$$
\left\{\begin{array}{l}
w_{0}^{h}+2\left(w_{1}^{h}+w_{2}^{h}+w_{3}^{h}\right)=1 \\
2 w_{1}^{h} h_{0}^{2}+2 w_{2}^{h}\left(2 h_{0}\right)^{2}+2 w_{3}^{h}\left(3 h_{0}\right)^{2}=1 \\
2 w_{1}^{h} h_{0}^{4}+2 w_{2}^{h}\left(2 h_{0}\right)^{4}+2 w_{3}^{h}\left(3 h_{0}\right)^{4}=3 \\
2 w_{1}^{h} h_{0}^{6}+2 w_{2}^{h}\left(2 h_{0}\right)^{6}+2 w_{3}^{h}\left(3 h_{0}\right)^{6}=15 \\
2 w_{1}^{h} h_{0}^{8}+2 w_{2}^{h}\left(2 h_{0}\right)^{8}+2 w_{3}^{h}\left(3 h_{0}\right)^{8}=105
\end{array}\right.
$$

After straightforward simplifications, we obtain the following explicit solution for the weights

$$
\left\{\begin{array}{l}
w_{3}^{h}=\left[h_{0}^{2}\left(4 h_{0}^{2}-15\right)+15\right] /\left(720 h_{0}^{6}\right), \\
w_{2}^{h}=\left[h_{0}^{2}\left(10-3 h_{0}^{2}\right)-5\right] /\left(40 h_{0}^{6}\right), \\
w_{1}^{h}=\left[h_{0}^{2}\left(12 h_{0}^{2}-13\right)+5\right] /\left(16 h_{0}^{6}\right), \\
w_{0}^{h}=1-2\left(w_{1}^{h}+w_{2}^{h}+w_{3}^{h}\right),
\end{array}\right.
$$

which is given in terms of the spacing parameter $h_{0}$. This spacing parameter is the unique positive solution of the equation

$$
\frac{12}{35} h_{0}^{6}-\frac{7}{5} h_{0}^{4}+2 h_{0}^{2}-1=0
$$

and has the approximate value

$$
h_{0} \approx 1.196979770393 .
$$

By direct calculation, one can verify that the quadrature weights specified by Eq. (29) are positive for this particular choice of $h_{0}$. The short-time propagation is then defined by the recurrence relation

$$
\psi_{\tau}^{\prime \prime}(x)=\sum_{j=-3}^{3} w_{j}^{h} r_{0}\left(x, x+j h_{0} \sigma ; \tau\right) \psi_{0}\left(x+j h_{0} \sigma\right)
$$

and has convergence order four provided that the continuous version of the short-time approximation has the same property. As for the short-time approximations of order two, one can argue that the optimal mesh is given by

$$
\Delta=h_{0} \sigma=h_{0}\left(\hbar^{2} \tau / m_{0}\right)^{1 / 2} .
$$


TABLE I: Parameters defining the short-time approximation of order 4 .

\begin{tabular}{|c|c|c|c|}
\hline$i$ & 1 & 2 & 3 \\
\hline$w_{i}$ & $1 / 3$ & $1 / 3$ & $1 / 3$ \\
\hline$\theta_{i}$ & $(2-\sqrt{2}) / 4$ & $1 / 2$ & $(2+\sqrt{2}) / 4$ \\
\hline$\tilde{\Lambda}_{1}\left(\theta_{i}\right)$ & 0 & $1 / 2$ & 0 \\
\hline$\tilde{\Lambda}_{2}\left(\theta_{i}\right)$ & $\sqrt{2} / 4$ & 0 & $\sqrt{2} / 4$ \\
\hline
\end{tabular}

The fourth-order short-time approximation we utilize for our numerical examples is the one recently described in Ref. 21. It has the form

$$
\begin{array}{r}
r_{0}\left(x, x^{\prime} ; \tau\right)=\left\{\int_{\mathbb{R}} d \mu\left(a_{1}\right) e^{-\tau w_{2} V\left[x_{r}\left(\theta_{2}\right)+\sigma a_{1} \tilde{\Lambda}_{1}\left(\theta_{2}\right)\right]}\right\} \\
\times\left\{\int_{\mathbb{R}} d \mu\left(a_{2}\right) e^{-\tau w_{1} V\left[x_{r}\left(\theta_{1}\right)+\sigma a_{2} \tilde{\Lambda}_{2}\left(\theta_{1}\right)\right]}\right. \\
\left.\times e^{-\tau w_{3} V\left[x_{r}\left(\theta_{3}\right)+\sigma a_{2} \tilde{\Lambda}_{2}\left(\theta_{3}\right)\right]}\right\},
\end{array}
$$

with the defining parameters enumerated in Table I. The quantity $x_{r}(u)$ is a straight line connecting the points $x$ and $x^{\prime}$, that is, $x_{r}(u)=x+\left(x^{\prime}-x\right) u$. Of course, as before, $\sigma=\left(\hbar^{2} \tau / m_{0}\right)^{1 / 2}$.

The approximation defined by Eq. (34) is part of a larger class of short-time approximations that have the property that they are positive and constructed from the potential function only. This class comprises approximations of the form ${ }^{22}$

$$
\begin{aligned}
& r_{0}^{(\nu)}\left(x, x^{\prime} ; \beta\right)=\int_{\mathbb{R}} d \mu\left(a_{1}\right) \ldots \int_{\mathbb{R}} d \mu\left(a_{n_{\nu}}\right) \\
& \times \exp \left\{-\beta \sum_{i=1}^{n_{q}} w_{i} V\left[x_{r}\left(\theta_{i}\right)+\sigma \sum_{k=1}^{n_{\nu}} a_{k} \tilde{\Lambda}_{k}\left(\theta_{i}\right)\right]\right\} .
\end{aligned}
$$

Here, $(\nu)$ denotes the order of convergence of the shorttime approximation. In addition to the issue of grid implementation, for such approximations, we are still left with the problem of specifying a minimalist quadrature technique for the integrals performed against the additional path variables, which are denoted by $a_{i}$. This problem is solved by the following theorem, the proof of which is deferred to Appendix 2.

Theorem 2 If a short-time/high-temperature approximation of the type expressed by Eq. (35) is replaced by

$$
\begin{aligned}
& r_{0}^{(\nu)}\left(x, x^{\prime} ; \beta\right)=\int_{\mathbb{R}} \ldots \int_{\mathbb{R}} d \mu_{c i}\left(a_{1}, \ldots, a_{n_{\nu}}\right) \\
& \times \exp \left\{-\beta \sum_{i=1}^{n_{q}} w_{i} V\left[x_{r}\left(\theta_{i}\right)+\sigma \sum_{k=1}^{n_{\nu}} a_{k} \tilde{\Lambda}_{k}\left(\theta_{i}\right)\right]\right\},
\end{aligned}
$$

then the order of convergence is preserved whenever the non-negative measure $d \mu_{c i}\left(a_{1}, \ldots, a_{n_{\nu}}\right)$ is centrally invariant and integrates exactly (as measured against the original Gaussian distribution) all polynomials in the variables $a_{1}, \ldots, a_{n_{\nu}}$ of degrees at most $2 \nu-1$.
By virtue of Theorem 2, the two-dimensional Gaussian integral appearing in Eq. (34) can be replaced with a $4 \times 4$ Gauss-Hermite cubature rule. However, the fourthorder short-time approximation expressed by Eq. (34) has the additional property that the variables $a_{1}$ and $a_{2}$ are independent. As such, each of the one-dimensional Gaussian measures can be replaced by a 4-point GaussHermite quadrature rule

$$
\begin{array}{r}
r_{0}\left(x, x^{\prime} ; \tau\right)=\left\{\sum_{j=1}^{4} w_{j}^{h} e^{-\tau w_{2} V\left[x_{r}\left(\theta_{2}\right)+\sigma z_{j}^{h} \tilde{\Lambda}_{1}\left(\theta_{2}\right)\right]}\right\} \\
\times\left\{\sum_{j=1}^{4} w_{j}^{h} e^{-\tau w_{1} V\left[x_{r}\left(\theta_{1}\right)+\sigma z_{j}^{h} \tilde{\Lambda}_{2}\left(\theta_{1}\right)\right]}\right. \\
\left.\times e^{-\tau w_{3} V\left[x_{r}\left(\theta_{3}\right)+\sigma z_{j}^{h} \tilde{\Lambda}_{2}\left(\theta_{3}\right)\right]}\right\} .
\end{array}
$$

To help the reader, we provide the actual values: $w_{1}^{h}=$ $w_{4}^{h}=0.25 /(3+\sqrt{6}), w_{2}^{h}=w_{3}^{h}=0.25 /(3-\sqrt{6})$, $z_{4}^{h}=-z_{1}^{h}=(3+\sqrt{6})^{1 / 2}$, and $z_{3}^{h}=-z_{2}^{h}=(3-\sqrt{6})^{1 / 2}$. Notice that the independence of the variables $a_{1}$ and $a_{2}$ brings some computational savings because the double integral over the variables $a_{1}$ and $a_{2}$ is really a product of one dimensional integrals. For $d$-dimensional systems, the quadrature is a product of $d$-dimensional integrals, each one requiring $4^{d}$ calls to the action. The total number of calls to the potential function is $3 \cdot 4^{d}$. In this respect, the reader can appreciate the superiority of the short-time approximation considered in Ref. 21 with regard to an older version presented in Ref. 22: the old version requires a number of $64^{d}$ calls to the potential function. As discussed at the end of Section II, GaussHermite quadrature techniques that utilize unevenly distributed quadrature knots may bring in important computational savings whenever the potential function is expensive to evaluate. For the definition of the short-time approximation, we are no longer constrained to utilize evenly distributed quadrature knots. Nevertheless, the numerical results presented in the following section do not utilize such advanced techniques. However, for $d \geq 3$, the preparation of the density matrix can easily become the more costly step and the utilization of polynomially scaling Gauss-Hermite quadrature schemes might be unavoidable.

\section{NUMERICAL EXAMPLES}

Given the relative complexity of the mathematical arguments presented, it is apparent that numerical verification of the results is necessary in order to get either confidence in the arguments or further insight into the capabilities of the techniques. Equally important, the high numerical accuracy afforded allows us to test the performance of the various imaginary-time approximations. The spatially continuous versions of these shorttime approximations are also utilized in Monte Carlo 
simulations. Nevertheless, due to the inherent statistical noise, it is virtually impossible to accurately quantify the quality of the short-time approximations from Monte Carlo simulations. All comparisons between the TrotterSuzuki and the fourth-order short-time approximations are made as if the computational effort were that characteristic for Monte Carlo simulations: the computational effort for one Metropolis step is three times larger for the fourth-order technique than for the Trotter-Suzuki approximation, for the same Trotter index. Because the fourth-order technique also leads to three times more path variables, this is equivalent to saying that the two approximations have the same computational effort for the same number of path variables.

\section{A. Numerical verification of the convergence order}

We study the convergence order of the Trotter-Suzuki and the fourth-order short-time approximations for the problem of computing the groundstate energy of two and three-dimensional coupled sextic oscillators. The technique employed is imaginary-time propagation of a delta function to a temperature low enough that the system practically resides in its groundstate. Although not the computationally most efficient one, the scheme utilized for extracting this information constitutes a thorough test on the overall quality and stability of the timepropagation scheme.

The potentials are those utilized by Kaluza ${ }^{23}$ to test his analytical Lanczos iteration method. For the two coupled sextic oscillators, the potential reads

$$
V(x, y)=V_{6}(x)+V_{6}(y)+x y,
$$

whereas for three coupled sextic oscillators, it has the form

$$
V(x, y, z)=V_{6}(x)+V_{6}(y)+V_{6}(z)+x y+x z+y z .
$$

The sextic-oscillator term is

$$
V_{6}(x)=\left(x^{2}+4 x^{4}+x^{6}\right) / 2 .
$$

Atomic units are utilized for all numerical results. The masses of the particles for the sextic-oscillator problem are all equal to 1 .

A grid representation of a delta function localized at the origin of the coordinate system is propagated to $\beta=20$, an inverse temperature for which both the two and three-dimensional systems reside in their groundstate within the numerical accuracy of the machine. The choice of the inverse temperature is based on the eigenenergies reported by Kaluza. As such, in one-dimensional notation, the groundstate density matrix is given by

$$
\rho(0, x ; \beta)=\psi_{g}(0) \psi_{g}(x) e^{-\beta E_{g}},
$$

from where we infer that

$$
E_{g}=-\frac{1}{\beta} \ln \left[\frac{\int_{\mathbb{R}} \rho(0, x ; \beta)^{2} d x}{\rho(0,0 ; \beta)}\right] .
$$

We mention that the systems have been localized in the boxes $[-6,6] \times[-6,6]$, for the two dimensional case, and $[-4,4] \times[-4,4] \times[-4,4]$, for the three-dimensional case. Dirichelet boundary conditions have been enforced. The boundaries are exactly those employed by Braun et al ${ }^{24}$ in testing their Chebyshev-Lanczos method.

The verification of the convergence order follows the following recipe. One evaluates

$$
E_{g, n}=-\frac{1}{\beta} \ln \left[\frac{\Delta \sum_{j=1}^{N} \rho_{n}\left(0, x_{j} ; \beta\right)^{2}}{\rho_{n}(0,0 ; \beta)}\right],
$$

for various Trotter indexes $n$, as well as the sequence

$\alpha_{m}=m^{2} \ln \left[1+\frac{E_{g, q m+p}-E_{g,(q+1) m+p}}{E_{g,(q+1) m+p}-E_{g}}\right], \quad m=1,2, \ldots$

Here $q$ and $p$, with $0 \leq p<q$, are integers specifying a given subsequence of $E_{g, 1}, E_{g, 2}, \ldots$. Any values $q$ and $p$ can be utilized with similar results: the slope of the sequence $\alpha_{m}$ converges to the actual order of the short-time approximation, which can be compared with the one predicted by theory. The choice of $q$ depends on the number of significant figures with which the energies are computed. Because the sequence of energies converges to the exact value $E_{g}$, the quantities $E_{g, q m+p}-E_{g,(q+1) m+p}$ can be very small numbers obtained as the difference of large ones. This is true especially of the techniques having faster convergence order. An appropriately large value for $q$ must be utilized. In the present application, we have employed the subsequence with $q=32$ and $p=0$. The exact groundstate energies are those reported by Kaluza: $E_{g}=1.992235763387$ for the two-dimensional case and $E_{g}=2.978302656837$ for the three-dimensional case, respectively.

The reader is also reminded that both $\Delta$ and $N$ in Eq. (42) actually depend on $n$. As discussed in the preceding sections, we have

$$
\Delta=h_{0} \sqrt{\frac{\hbar^{2} \beta}{m_{0} n}}
$$

with $h_{0}=\sqrt{3}$ for second-order short-time approximations and $h_{0} \approx 1.196979770393$ for fourth-order approximations. In addition,

$$
N=1+\left[\frac{b-a}{\Delta}\right]
$$

with $[x]$ denoting the largest integer smaller or equal to $x$. Because the number of discretization points varies discontinuously, certain oscillations might appear in the slopes of $\alpha_{m}$, for small values of $m$. Nonetheless, these oscillations must disappear after the asymptotic regime sets in.

As shown in Figs. 1 and 2, the predicted theoretical values for the convergence orders of the spatially-discretized Trotter-Suzuki and fourth-order short-time approximations are well supported by the actual numerical data. 


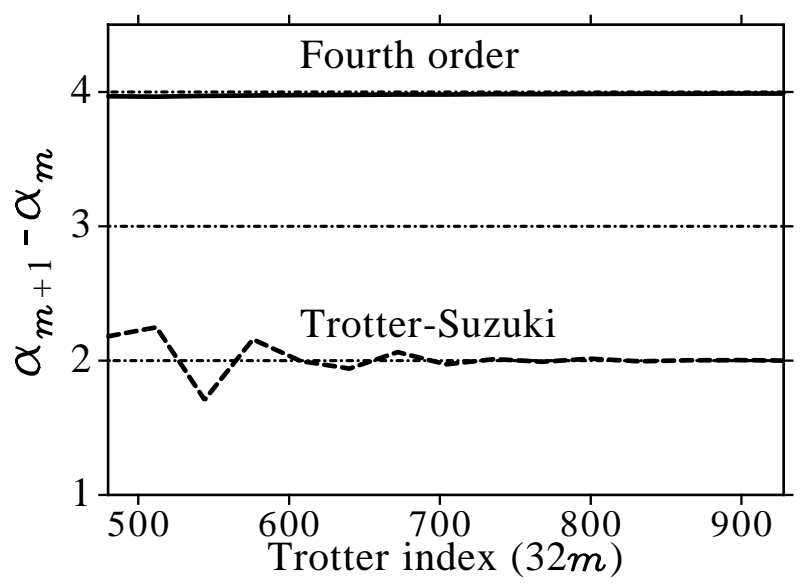

FIG. 1: Instantaneous order of convergence as a function of the Trotter index for the two-dimensional sextic oscillator. The oscillations that appear for small Trotter indexes are due to the discontinuous change in the number of grid points. The oscillations for the fourth-order short-time approximation do not appear in this range of Trotter indexes because of the denser grid.

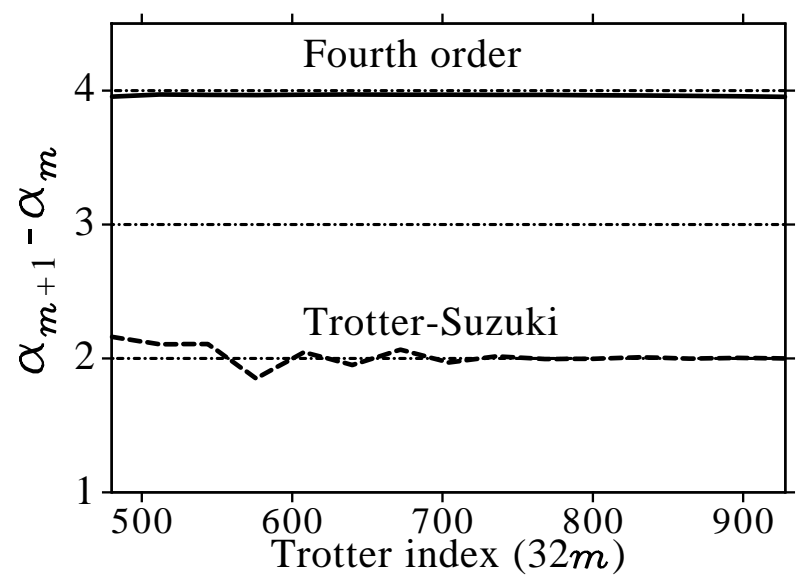

FIG. 2: Same as in Fig. 1, but for the three-dimensional sextic oscillator. The performance of the fourth-order technique is not affected by the increase in dimensionality.

Indirectly, these data also support the assertion that the original spatially-continuous short-time approximations have the same convergence order as their discretized counterparts (given the way the latter are derived).

For Monte Carlo simulations, the Trotter-Suzuki approximation requires $n+1$ calls to the potential function, whereas the fourth order approximation requires $3 n$ calls (see Eq. 34) for a Trotter index equal to $n$. Therefore, for the same computational effort, the former approximation can afford Trotter indexes that are three times larger. Table II thus records the actual estimates to the groundstate energies together with their difference from the essentially exact values reported by Kaluza. Also presented are the grid sizes employed. One sees that for the problem of computing groundstate energies by propagation in imaginary time, the computational savings obtained by employing the fourth-order short-time approximation are rather modest, with factors between 2.5 and 3.0. Nevertheless, in Monte Carlo simulations, the energy is evaluated in a different way, via estimators obtained by differentiation of the partition function. As we shall see in the last part of the present section, the computational savings obtained by employing the fourthorder short-time approximation are more significant for those quantities that are obtained by differentiation of the density matrix.

\section{B. Higher-energy eigenfunctions}

A faster approach to obtaining groundstate energies and eigenfunctions is provided by the popular Lanczos iteration. ${ }^{25}$ This approach is essentially a Gram-Schmidt orthogonalization procedure applied to the Krylov space, which is obtained recursively by application of the density matrix to a randomly generated initial function. We have tested this approach and obtained convergence of the energies to within machine accuracy after 15 to 30 iterations in most cases. Unfortunately, the energies for the excited states were quite in error, due to the numerical instability of the Lanczos iteration. This numerical instability can be overcome by restarting the iteration in the subspace orthogonal on the previously determined eigenfunctions. The explicit enforcement of orthogonality implies that the cost increases as the square of the number of eigenfunctions to be determined. Nevertheless, techniques such as the block-Lanczos method with selective orthogonalization ${ }^{26}$ can control the linear independence of the Lanczos vectors without the cost of full orthogonalization. This technique has been employed by Braun et $a l^{24}$ to compute eigenvalues of the examples presented here and some other with very high accuracy. They have utilized a Chebyshev approximation to the density matrix, which is more accurate, although computationally more expensive than the local methods presented here (for the same grid size). Based on their experience, one can safely infer that the block-Lanczos method with selective orthogonalization will find its usefulness together with the present approximations as well. The main reason is that the density matrix is utilized solely for the generation of the Lanczos vectors and one can fully exploit the natural sparsity of the matrix-vector multiplication characteristic of the spatially-discretized short-time approximations.

Nevertheless, in order to quantify the quality of the short-time approximations presented, it appears safer to directly diagonalize the short-time approximation by use of algorithms that employ orthogonal transformations to do so. For one-dimensional systems, this work is facilitated by the fact that the density matrix is banddiagonal, with a number of distinct codiagonals equal to 
TABLE II: Estimates of the groundstate energies for the two and three dimensional sextic oscillators. The results in each line are computed with different Trotter indexes for the two different short-time approximations, commensurate with the computational effort in a Monte Carlo simulation. In each case and for each approximation, we have determined the minimal Trotter index that guarantees three significant digits. A ratio measuring the Monte Carlo computational effort necessary to reach this precision is computed.

\begin{tabular}{|c|c|c|c|c|c|c|c|}
\hline Trotter index & grid size & energy & difference & Trotter index & grid size & energy & difference \\
\hline \multicolumn{4}{|c|}{ Trotter-Suzuki } & \multicolumn{4}{|c|}{ fourth-order } \\
\hline \multicolumn{8}{|c|}{ two dimensional sextic oscillator } \\
\hline 192 & $22 \times 22$ & 1.975109442 & $-.1710^{-1}$ & 64 & $18 \times 18$ & 1.989624232 & $-.2610^{-2}$ \\
\hline 384 & $31 \times 31$ & 1.987692851 & $-.4510^{-2}$ & 128 & $26 \times 26$ & 1.991597326 & $-.6410^{-3}$ \\
\hline 768 & $43 \times 43$ & 1.991121783 & $-.1110^{-2}$ & 256 & $36 \times 36$ & 1.992201798 & $-.3410^{-4}$ \\
\hline \multicolumn{8}{|c|}{ MC computational effort: $812:(3 \cdot 104)=2.60: 1$} \\
\hline \multicolumn{8}{|c|}{ three dimensional sextic oscillator } \\
\hline 192 & $15 \times 15 \times 15$ & 2.948281864 & $-.3010^{-1}$ & 64 & $12 \times 12 \times 12$ & 2.974739961 & $-.3610^{-2}$ \\
\hline 384 & $21 \times 21 \times 21$ & 2.971411405 & $-.6910^{-2}$ & 128 & $17 \times 17 \times 17$ & 2.978042841 & $-.2610^{-3}$ \\
\hline 768 & $29 \times 29 \times 29$ & 2.976612654 & $-.1710^{-2}$ & 256 & $24 \times 24 \times 24$ & 2.978250681 & $-.5210^{-4}$ \\
\hline \multicolumn{8}{|c|}{ MC computational effort: $996:(3 \cdot 116)=2.86: 1$} \\
\hline
\end{tabular}

1 for the Trotter approximation and to 3 for the fourthorder approximation. For example, in the case of the Trotter approximation, the main diagonal is made up of the values $w_{0}^{h} r_{0}\left(x_{i}, x_{i} ; \tau\right)$, whereas the codiagonal is composed of the values $w_{1}^{h} r_{0}\left(x_{i}, x_{i+1} ; \tau\right)$. The other codiagonal, which formally is given by $w_{-1}^{h} r_{0}\left(x_{i+1}, x_{i} ; \tau\right)$, is identical to the first by symmetry. The IMSL routine ${ }^{27}$ we have utilized transforms a band diagonal matrix into a tridiagonal one (if it is not already so) by orthogonal similarity transformations. The tridiagonal matrix is then diagonalized by the rational QR algorithm with Newton corrections.

In the limit of small inverse temperatures $\tau$, the eigenvalues of the short-time approximation take the form

$$
\exp \left(-\tau E_{k}\right)
$$

where the $E_{k}$ 's are the eigenvalues of the Hamiltonian. As $\tau \rightarrow 0$, the eigenvalues of the density matrix converge to 1 and become degenerate. Unfortunately, $\tau \rightarrow 0$ is also the limit in which the short-time approximations become exact. Nevertheless, the resolution capabilities of the eigenvalue solver were not exceeded for the values of $\tau$ employed in the following numerical demonstration, despite the additional difficulties arising from the fact that the energy eigenvalues are very close one to each other. The example studied is the Morse potential for the $\mathrm{I}_{2}$ molecule, which is given by the equation ${ }^{24,28}$

$$
V(x)=D[\exp (-\alpha x)-1]^{2},
$$

with $D=0.0224$ a.u., $\alpha=0.9374$ a.u., and a reduced mass $m_{0}=119406$ a.u. The eigenvalues are given analytically by the formula ${ }^{29}$

$$
E_{\nu}=5.741837286 \cdot 10^{-4}\left[\nu+\frac{1}{2}-\frac{1}{\xi}\left(\nu+\frac{1}{2}\right)^{2}\right] \text { a.u. }
$$

with $\xi=156.047612535$ and $\nu$ a non-negative integer.

The inverse temperature $\tau$ is chosen such that the number of grid points in the interval $[-1,3]$ is 128 , for the fourth-order approximation. This choice of parameters enables us to compare the performance of the fourth-order short-time approximation with that of the Chebyshev approximation. We have also diagonalized the Trotter-Suzuki approximation for a value of $\tau$ three times smaller, which corresponds to a number of grid points equal to 153 . This facilitates a direct comparison with the fourth-order technique for the same Monte Carlo effort. While individual eigenvalues are next to impossible to compute by path integral Monte Carlo techniques, their exactness is relevant of the quality of the simulation, because they enter the expressions of all thermodynamic properties in the form of Boltzmann sums.

A look at Table III shows that the fourth order approximation is capable of producing accuracies ranging from 8 digits, for the lower energies, to 5 digits for the larger energies. The accuracy of the Trotter-Suzuki approximation ranges from 5 to about 3 digits, in agreement with its lower convergence order. By comparison, the Chebyshev approximation utilized by Braun $e t a l^{24}$ is more capable. Its accuracy remains virtually unchanged, at about 8 digits. Nevertheless, although they converge only polynomially, the spatially-discretized short-time approximations have their own strengths, being significantly faster for the same grid size and more advantageous for larger dimensional systems. As always when comparing global versus local methods, the first ones turn out to be more useful if high precision is desired, whereas the second ones can tackle larger systems under a wider range of boundary conditions. 
TABLE III: Lowest energy eigenvalues of the Morse oscillator for the $\mathrm{I}_{2}$ molecule.

\begin{tabular}{|c|c|c|c|c|c|}
\hline$\nu$ & analytical result & fourth-order $(N=128)$ & difference & Trotter-Suzuki $(N=153)$ & difference \\
\hline 0 & $.28617197910^{-03}$ & $.28617197110^{-03}$ & $-.7910^{-11}$ & $.28616680510^{-03}$ & $-.5210^{-08}$ \\
\hline 1 & $.85299662410^{-03}$ & $.85299659510^{-03}$ & $-.2910^{-10}$ & $.85297290910^{-03}$ & $-.2410^{-07}$ \\
\hline 2 & $.14124621810^{-02}$ & $.14124621110^{-02}$ & $-.7510^{-10}$ & $.14123955710^{-02}$ & $-.6710^{-07}$ \\
\hline 3 & $.19645686610^{-02}$ & $.19645684710^{-02}$ & $-.2010^{-09}$ & $.19644201810^{-02}$ & $-.1510^{-06}$ \\
\hline 4 & $.25093160610^{-02}$ & $.25093155710^{-02}$ & $-.4910^{-09}$ & $.25090335710^{-02}$ & $-.2810^{-06}$ \\
\hline 5 & $.30467043610^{-02}$ & $.30467032710^{-02}$ & $-.1110^{-08}$ & $.30462240210^{-02}$ & $-.4810^{-06}$ \\
\hline 6 & $.35767335910^{-02}$ & $.35767313510^{-02}$ & $-.2210^{-08}$ & $.35759812110^{-02}$ & $-.7510^{-06}$ \\
\hline 7 & $.40994037310^{-02}$ & $.40993995210^{-02}$ & $-.4210^{-08}$ & $.40982961710^{-02}$ & $-.1110^{-05}$ \\
\hline 8 & $7910^{-02}$ & $.46147073910^{-02}$ & $-.7410^{-08}$ & $.46131612810^{-02}$ & $-.1610^{-05}$ \\
\hline 9 & $.51226667710^{-02}$ & $.51226545110^{-02}$ & $-.1210^{-07}$ & $.51205702410^{-02}$ & $-.2110^{-05}$ \\
\hline 10 & $.56232596610^{-02}$ & $.56232403510^{-02}$ & $-.1910^{-07}$ & $.56205179810^{-02}$ & $-.2710^{-05}$ \\
\hline 11 & $.61164934610^{-02}$ & $.61164642710^{-02}$ & $-.2910^{-07}$ & $61130006910^{-02}$ & $-.3510^{-05}$ \\
\hline 12 & $.66023681910^{-02}$ & $.66023256010^{-02}$ & $-.4310^{-07}$ & $.65980157410^{-02}$ & $-.4410^{-05}$ \\
\hline 13 & $.70808838210^{-02}$ & $.70808235710^{-02}$ & $-.6010^{-07}$ & $.70755616210^{-02}$ & $-.5310^{-05}$ \\
\hline 14 & $.75520403810^{-02}$ & $.75519573610^{-02}$ & $-.8310^{-07}$ & $.75456379610^{-02}$ & $-.6410^{-05}$ \\
\hline 15 & $.80158378510^{-02}$ & $.80157261110^{-02}$ & $-.1110^{-06}$ & $.80082454410^{-02}$ & $-.7610^{-05}$ \\
\hline 16 & $.84722762410^{-02}$ & $.84721289210^{-02}$ & $-.1510^{-06}$ & $.84633857410^{-02}$ & $-.8910^{-05}$ \\
\hline 17 & $410^{-02}$ & $.89211648810^{-02}$ & $-.1910^{-06}$ & $.89110615110^{-02}$ & $-.1010^{-04}$ \\
\hline 18 & $10^{-02}$ & $.93628330610^{-02}$ & $-.2410^{-06}$ & $.93512763410^{-02}$ & $-.1210^{-04}$ \\
\hline 19 & $.97974369010^{-02}$ & $.97971325510^{-02}$ & $-.3010^{-06}$ & $.97840346410^{-02}$ & $-.1310^{-04}$ \\
\hline 20 & $010^{-01}$ & $.10224062510^{-01}$ & $-.3810^{-06}$ & $.10209341710^{-01}$ & $-.1510^{-04}$ \\
\hline 21 & $.10644081910^{-01}$ & $.10643622010^{-01}$ & $-.4610^{-06}$ & $.10627203510^{-01}$ & $-.1710^{-04}$ \\
\hline 22 & $.11056365810^{-01}$ & $.11055810310^{-01}$ & $-.5610^{-06}$ & $.11037626810^{-01}$ & $-.1910^{-04}$ \\
\hline 23 & $.11461290610^{-01}$ & $.11460626610^{-01}$ & $-.6610^{-06}$ & $.11440619110^{-01}$ & $-.2110^{-04}$ \\
\hline 24 & $.11858856310^{-01}$ & $.11858070510^{-01}$ & $-.7910^{-06}$ & $.11836188210^{-01}$ & $-.2310^{-04}$ \\
\hline
\end{tabular}

\section{Thermodynamic data involving temperature derivatives}

As pointed out by Yamamoto, ${ }^{30}$ different thermodynamic properties and estimators may exhibit different sensitivities to the quality of the short-time approximation. We have seen that the fourth-order approximation has a relatively modest computational advantage over the Trotter-Suzuki approximation for the problem of computing groundstate energies of sextic oscillators by means of Eq. (41). A same modest improvement (a factor between 2.5 and 3.0) is expected if the energy is evaluated by means of the formula

$$
E_{g}=-\beta^{-1} \ln [Z(\beta)],
$$

where $Z(\beta)$ is the partition function and $\beta$ corresponds to a low enough temperature. One of the numerically equivalent ways of computing the partition function is to diagonalize the short-time approximation for the inverse temperature $\tau_{n}=\beta / n$, raise the eigenvalues to the power $n$, and take the trace. Of course, we apply here the invariance of the trace with respect to a change of basis. We obtain

$$
Z(\beta)=\sum_{k} e^{-\beta E_{k}\left(\tau_{n}\right)}
$$

where the notation employed shows explicitly that the estimates for the energy eigenvalues are actually dependent on $\tau_{n}$, therefore on $\beta$. It is only in the limit $\tau_{n} \rightarrow 0$ that the true eigenvalues are recovered

$$
E_{k}=\lim _{n \rightarrow \infty} E_{k}\left(\tau_{n}\right)=\lim _{\tau \rightarrow 0} E_{k}(\tau) .
$$

Other thermodynamic quantities, such as the average energy and the heat capacity, can be obtained from the spectral decomposition by means of the formulas

$$
\langle E\rangle_{\beta}^{(1)}=\frac{\sum_{k} e^{-\beta E_{k}\left(\tau_{n}\right)} E_{k}\left(\tau_{n}\right)}{\sum_{k} e^{-\beta E_{k}\left(\tau_{n}\right)}}
$$

and

$$
\begin{aligned}
\left\langle C_{V}\right\rangle_{\beta}^{(1)}= & k_{B} \beta^{2}\left\{\frac{\sum_{k} e^{-\beta E_{k}\left(\tau_{n}\right)} E_{k}\left(\tau_{n}\right)^{2}}{\sum_{k} e^{-\beta E_{k}\left(\tau_{n}\right)}}\right. \\
& \left.-\left[\frac{\sum_{k} e^{-\beta E_{k}\left(\tau_{n}\right)} E_{k}\left(\tau_{n}\right)}{\sum_{k} e^{-\beta E_{k}\left(\tau_{n}\right)}}\right]^{2}\right\},
\end{aligned}
$$

respectively. At first sight, we could not expect any major gain in computational efficiency of the fourth-order approximation over the Trotter-Suzuki approximation, other than the one already documented. After all, the 
expressions above are well-behaved functionals of the energy eigenvalues.

However, in Monte Carlo simulations, the average energy and the heat capacity are not evaluated this way. Rather, the Monte Carlo estimators are obtained by differentiation of the partition function. More precisely, the average energy is evaluated from the formula

$$
\langle E\rangle_{\beta}^{(2)}=-\frac{\partial}{\partial \beta} \ln [Z(\beta)],
$$

whereas the heat capacity is obtained from

$$
\begin{aligned}
& \left\langle C_{V}\right\rangle_{\beta}^{(2)}=k_{B} \beta^{2} \frac{\partial^{2}}{\partial \beta^{2}} \ln [Z(\beta)] \\
& \approx \frac{k_{B}}{\epsilon^{2}} \ln \left\{\frac{Z[\beta(1+\epsilon)] Z[\beta(1-\epsilon)]}{Z(\beta)^{2}}\right\} .
\end{aligned}
$$

As the second part of the last equation suggests, the differentiation can be performed numerically, by finite difference. A value of $\epsilon=2^{-8}$ proves capable of providing at least 3 exact digits for the sextic oscillator, enough for our purposes.

Are Eqs. (52) and (53) computationally equivalent to (54) and (55)? The unfortunate answer is no. We say unfortunate because the spectral estimators denoted by the superscript (1) are better behaved than the Monte Carlo estimators denoted by (2). For the energy, the relationship between the Monte Carlo and the spectral estimators is given by

$$
\langle E\rangle_{\beta}^{(2)}=\langle E\rangle_{\beta}^{(1)}+\tau_{n} \frac{\sum_{k} e^{-\beta E_{k}\left(\tau_{n}\right)} E_{k}^{\prime}\left(\tau_{n}\right)}{\sum_{k} e^{-\beta E_{k}\left(\tau_{n}\right)}},
$$

where

$$
E_{k}^{\prime}(\tau)=\frac{d}{d \tau} E_{k}(\tau)
$$

denotes the derivative of the $k$-th eigenvalue with respect to $\tau$. This derivative is zero for the exact Hamiltonian eigenvalues, which are temperature independent, but not for the eigenvalues obtained from a short-time approximation. A similar state of affairs holds for the Monte Carlo heat capacity estimator, for which the second order derivative introduces additional bias over the spectral estimator.

The numerical evidence presented in the first part of the present section for the groundstate energy of the sextic oscillators supports the theoretical prediction that, as is the case for other properties, the convergence of the eigenvalues is as fast as $O\left(1 / n^{\nu}\right)=O\left(\tau_{n}{ }^{\nu}\right)$, where $\nu$ is the convergence order. That is,

$$
E_{k}\left(\tau_{n}\right)=E_{k}+O\left(\tau_{n}{ }^{\nu}\right),
$$

from which one quickly sees that only the derivatives up to order $\nu-1$ vanish in the limit $\tau_{n} \rightarrow 0$. The derivatives of order $\nu$ may converge to some non-zero quantity or, more generally, only be bounded. The higher order
TABLE IV: Heat capacities for the one-dimensional sextic oscillator in units of the Boltzmann constant $k_{B}$. For each short-time approximation, the Trotter index was increased until the relative error for the Monte Carlo heat capacity estimator became about $1 \%$. The superscripts (2) and (1) denote the Monte Carlo and the spectral estimators, respectively.

\begin{tabular}{|c|c|c|c|c|}
\hline Trotter index & $\left\langle C_{V}\right\rangle_{\beta}^{(2)}$ & error & $\left\langle C_{V}\right\rangle_{\beta}^{(1)}$ & error \\
\hline \multicolumn{6}{|c|}{ Trotter-Suzuki } \\
\hline 1,000 & $.11510^{-2}$ & $208 \%$ & $.55310^{-3}$ & $0.0 \%$ \\
2,500 & $.64910^{-3}$ & $17.4 \%$ & $.55310^{-3}$ & $0.0 \%$ \\
5,000 & $.57710^{-3}$ & $4.3 \%$ & $.55310^{-3}$ & $0.0 \%$ \\
10,000 & $.55910^{-3}$ & $1.1 \%$ & $.55310^{-3}$ & $0.0 \%$ \\
\hline \multicolumn{6}{|c|}{ fourth-order } \\
\hline 100 & $.88410^{-3}$ & $59.9 \%$ & $.55310^{-3}$ & $0.0 \%$ \\
150 & $.61710^{-3}$ & $11.6 \%$ & $.55310^{-3}$ & $0.0 \%$ \\
225 & $.56710^{-3}$ & $2.5 \%$ & $.55310^{-3}$ & $0.0 \%$ \\
300 & $.55810^{-3}$ & $0.9 \%$ & $.55310^{-3}$ & $0.0 \%$ \\
\hline MC computational effort: $10,000:(3 \cdot 300) \approx 11: 1$ \\
\hline
\end{tabular}

derivatives diverge. Fortunately, the thermodynamic estimators only contain terms of the type $\tau^{q} \partial^{q} E_{k} / \partial \tau^{q}$ evaluated for $\tau=\tau_{n}$. They converge to 0 as fast as $O\left(\tau_{n}{ }^{\nu}\right)$, whether the individual derivatives of order $q$ converge or diverge. As such, the biasing term in Eq. (56) goes to zero as fast as $O\left(\tau_{n}^{2}\right)$ for the Trotter-Suzuki approximation and as fast as $O\left(\tau_{n}{ }^{4}\right)$ for the fourth-order approximation. In other words the overall convergence order is not affected. Nevertheless, the convergence constant for the energy and heat capacity estimators designated by the superscripts (1) and (2) are quite different, a fact that we are going to demonstrate by studying numerically the errors for the heat capacity.

We consider the one-dimensional sextic potential of Kaluza, which is given by Eq (40). At the inverse temperature $\beta=5$, the system is virtually in its groundstate, with a minute contribution from the next excited state of about 3.5 parts in one million. Within the simple picture of local harmonic analysis, the practical relevance of studying a system at such a low temperature is as follows. It is often the case that different normal modes have quite different force constants. As such, a few of the modes may contain several excited states, whereas most others might be practically in their groundstate. The presence of the first kind means that quite interesting physics is still taking place, perhaps to be noticed by monitoring the heat capacity. Unfortunately, the presence of the others means that getting access to this physics is a difficult computational task. Although their individual contribution to the heat capacity is small, the latter kind of local minima represent the major source of both statistical and systematic errors. Here, we quantify the second type of errors.

The results in Table IV speak for themselves. For the ranges of Trotter indexes needed to reasonably converge the Monte Carlo estimators, the estimators ob- 
tained from spectral analysis are fully converged. This means that the biasing terms arising from the dependence of the eigenfunctions on the inverse temperature represent the major source of systematic errors. The fourth-order short-time approximation addresses the convergence problems of the Monte Carlo estimators in a more successful manner than the Trotter-Suzuki approximation. In fact, for this particular system, the former technique is about 11 times faster. The need for shorttime approximations having faster asymptotic convergence will be even greater for the problem of computing moments of spectral functions, ${ }^{31,32}$ where derivatives of very high order are evaluated. At the same time, one cannot help but wonder how the Monte Carlo estimators should be modified so that to circumvent the influence of the dependence of the eigenvalues on the inverse temperature, which is conducive of large systematic errors. Nevertheless, the use of short-time approximations having higher convergence order is definitely a winning strategy.

\section{SUMMARY AND CONCLUSIONS}

For real-time propagation, the Lie-Trotter construction is sufficiently powerful to provide unitary short-time approximations of any polynomial order. However, as the Suzuki non-existence theorem shows, for imaginarytime propagation, stable and positive Lie-Trotter hightemperature approximations of order greater or equal to 3 are not available. Nevertheless, there exist a large array of more general high-temperature approximations that can be utilized in a Lie-Trotter product form. The convergence of such products is guaranteed by the FeynmanKac formula. Such high-temperature approximations can be spatially discretized with preservation of both the positivity and the convergence order of the original approximations. The result is a sparse density matrix, which is actually band-diagonal for one-dimensional systems. The sparsity of the density matrix is $3^{d}$ for approximations having order of convergence 2 and $7^{d}$ for the approximations having order of convergence 4. Theoretically, this sparsity can be further improved by utilizing polynomially scaling $d$-dimensional Gauss-Hermite cubature techniques, perhaps at the cost of introducing negative weights. Techniques of interpolation may in principle be utilized to cope with the irregularity of the cubature knots. The usefulness of such approaches remains to be investigated.

The Monte Carlo estimators derived by temperature differentiation of the density matrix or related quantities exhibit a systematic bias when compared with their spectral counterparts. This bias is due to the temperature dependence of the energy eigenvalues obtained from the short-time approximation. It generally decays to zero at a rate dictated by the convergence order of the approximation. For the estimators obtained by temperature differentiation, the fourth-order high-temperature approximation allows for significant computational savings over the Trotter-Suzuki counterpart. For those properties that do not involve differentiation of the density matrix, the Monte Carlo and the spectral estimators coincide. In such cases, the computational advantage of the fourth-order approximation over the Trotter-Suzuki counterpart is not as pronounced.

\section{Acknowledgments}

This work was supported in part by the National Science Foundation Grant No. CHE-0345280 and by the Director, Office of Science, Office of Basic Energy Sciences, Chemical Sciences, Geosciences, and Biosciences Division, U.S. Department of Energy under Contract No. DE-AC02-05CH11231.

\section{APPENDIX A: PROOF OF THEOREM 1}

To begin with, consider a positive short-time approximation $\rho_{0}\left(x, x^{\prime} ; \beta\right)$ as well as the Lie-Trotter product that it generates

$$
\begin{array}{r}
\rho_{n}\left(x, x^{\prime} ; \beta\right)=\int_{\mathbb{R}} d x_{1} \ldots \int_{\mathbb{R}} d x_{n-1} \rho_{0}\left(x, x_{1} ; \tau_{n}\right) \\
\ldots \rho_{0}\left(x_{n-1}, x^{\prime} ; \tau_{n}\right),
\end{array}
$$

where $\tau_{n}=\beta / n$. Assume that there exists the Hermitian operator $T_{\nu}$, called a convergence operator, that associates to each infinitely differentiable and compactly supported function $\phi(x)$ the square integrable function

$$
\left(T_{\nu} \phi\right)(x)=\lim _{\tau \rightarrow 0^{+}} \frac{\int_{\mathbb{R}}\left[\rho_{0}\left(x, x^{\prime} ; \tau\right)-\rho\left(x, x^{\prime} ; \tau\right)\right] \phi\left(x^{\prime}\right) d x^{\prime}}{\tau^{\nu+1}} .
$$

A recently introduced conjecture on the convergence of Lie-Trotter products ${ }^{22}$ asserts that the convergence of the Lie-Trotter product is $O\left(1 / n^{\nu}\right)$. More strongly,

$$
\begin{aligned}
& \lim _{n \rightarrow \infty} n^{\nu}\left[\rho_{n}\left(x, x^{\prime} ; \beta\right)-\rho\left(x, x^{\prime} ; \beta\right)\right]=\beta^{\nu+1} \\
& \quad \times \int_{0}^{1}\left\langle x\left|e^{-\theta \beta H} T_{\nu} e^{-(1-\theta) \beta H}\right| x^{\prime}\right\rangle d \theta .
\end{aligned}
$$

For the problem of propagating a square-integrable function $\psi_{0}(x)$ in imaginary time, if $\psi_{\beta}^{(n)}(x)$ represents the $n$-fold iteration of the algorithm specified by Eq. (7), then

$$
\begin{gathered}
\psi_{\beta}^{(n)}(x)=\psi_{\beta}(x)+\frac{\beta^{\nu+1}}{n^{\nu}} \int_{\mathbb{R}} d x^{\prime} \int_{0}^{1} d \theta \psi_{0}\left(x^{\prime}\right) \\
\times\left\langle x\left|e^{-\theta \beta H} T_{\nu} e^{-(1-\theta) \beta H}\right| x^{\prime}\right\rangle+O\left(1 / n^{\nu+1}\right) .
\end{gathered}
$$

Eq. (A2) says that the asymptotic convergence predicted by Eq. (A4) happens provided that

$$
\phi_{\tau}^{\prime}(x)=\phi_{\tau}(x)+O\left(\tau^{\nu+1}\right)
$$


for all infinitely differentiable and square integrable functions $\phi_{0}(x)$. Here,

$$
\begin{aligned}
\phi_{\tau}(x) & =\int_{\mathbb{R}} \rho\left(x, x^{\prime} ; \tau\right) \phi_{0}\left(x^{\prime}\right) d x^{\prime} \\
& =\int_{\mathbb{R}} r(x, x+z \sigma ; \tau) \phi_{0}(x+z \sigma) d \mu(z)
\end{aligned}
$$

represents the exactly-propagated wavefunction, whereas

$$
\begin{aligned}
\phi_{\tau}^{\prime}(x) & =\int_{\mathbb{R}} \rho_{0}\left(x, x^{\prime} ; \tau\right) \phi_{0}\left(x^{\prime}\right) d x^{\prime} \\
& =\int_{\mathbb{R}} r_{0}(x, x+z \sigma ; \tau) \phi_{0}(x+z \sigma) d \mu(z)
\end{aligned}
$$

represents the wavefunction propagated through the short-time approximation. In words, what dictates the asymptotic convergence is the degree by which $\phi_{\tau}^{\prime}(x)$ and $\phi_{\tau}(x)$ differ in the short-time limit.

Let us replace the Gaussian measure $d \mu(z)$ appearing in Eq. (A7) with some other positive distribution $d \mu_{0}(z)$ and let

$$
\phi_{\tau}^{\prime \prime}(x)=\int_{\mathbb{R}} r_{0}(x, x+z \sigma ; \tau) \phi_{0}(x+z \sigma) d \mu_{0}(z) .
$$

If the difference between $\phi_{\tau}^{\prime \prime}(x)$ and $\phi_{\tau}^{\prime}(x)$ is also of order $O\left(\tau^{\nu+1}\right)$, then the difference between $\phi_{\tau}^{\prime \prime}(x)$ and $\phi_{\tau}(x)$ is still of order $O\left(\tau^{\nu+1}\right)$. Therefore, by virtue of Eq. (A5), in order to demonstrate Theorem 1, it is enough to prove that the difference between $\phi_{\tau}^{\prime \prime}(x)$ and $\phi_{\tau}^{\prime}(x)$ is of order $O\left(\tau^{\nu+1}\right)$.

Define

$$
D_{k}(x ; \tau)=\left.\frac{d^{k}}{d x^{\prime}} r_{0}\left(x, x^{\prime} ; \tau\right) \psi_{0}\left(x^{\prime}\right)\right|_{x^{\prime}=x}
$$

and notice (by Taylor expansion) that

$$
\phi_{\tau}^{\prime}(x)=\sum_{k=0}^{2 \nu+1} \frac{\sigma^{k}}{k !} D_{k}(x ; \tau)\left[\int_{\mathbb{R}} z^{k} d \mu(z)\right]+O\left(\sigma^{2 \nu+2}\right)
$$

and

$$
\phi_{\tau}^{\prime \prime}(x)=\sum_{k=0}^{2 \nu+1} \frac{\sigma^{k}}{k !} D_{k}(x ; \tau)\left[\int_{\mathbb{R}} z^{k} d \mu_{0}(z)\right]+O\left(\sigma^{2 \nu+2}\right) .
$$

Because $\sigma=\left(\hbar^{2} \tau / m_{0}\right)^{1 / 2}$, it follows that $O\left(\sigma^{2 \nu+2}\right)=$ $O\left(\tau^{\nu+1}\right)$. Thus, the left-hand sides of Eqs. (A9) and (A10) agree to an accuracy of $O\left(\tau^{\nu+1}\right)$ if and only if

$$
\int_{\mathbb{R}} z^{k} d \mu(z)=\int_{\mathbb{R}} z^{k} d \mu_{0}(z), \quad \forall 0 \leq k \leq 2 \nu+1 .
$$

The proof of Theorem 1 is concluded.

\section{APPENDIX B: PROOF OF THEOREM 2}

We begin the proof of Theorem 2 by recording a convergence result from Ref. 22. A short-time approximation of the type given by Eq. (35) has convergence order $\nu$ if and only if

$$
\begin{aligned}
& \mathbb{E}\left[\left(B_{1}\right)^{j_{1}}\left(M_{0}\right)^{j_{2}}\left(M_{1}\right)^{j_{3}} \ldots\left(M_{2 m-2}\right)^{j_{2 m}}\right] \\
& =\mathbb{E}\left[\left(\tilde{B}_{1}\right)^{j_{1}}\left(\tilde{M}_{0}\right)^{j_{2}}\left(\tilde{M}_{1}\right)^{j_{3}} \ldots\left(\tilde{M}_{2 m-2}\right)^{j_{2 m}}\right]
\end{aligned}
$$

for all $2 m$-tuples of non-negative integers $\left(j_{1}, j_{2}, \ldots, j_{2 m}\right)$ such that

$$
\sum_{k=1}^{2 m} k j_{k}=2 m
$$

and $1 \leq m \leq \nu$. The left-hand side expression involves the expectations of certain functionals of a standard Brownian motion. The relevant definitions can be found in the aforementioned reference and are not important for the present development. The right-hand side expression involves the quantities $\tilde{B}_{1}=z$ as well as

$$
\tilde{M}_{k}=\sum_{i=1}^{n_{q}} w_{i}\left[z \theta_{i}+\sum_{j=1}^{n_{\nu}} a_{j} \tilde{\Lambda}_{j}\left(\theta_{i}\right)\right]^{k}
$$

which are functions of the random variables $z, a_{1}, \ldots, a_{n_{\nu}}$ (in the original work, $z$ was denoted by $a_{0}$ ). These variables are assumed to be independent and identically distributed normal random variables. In other words, the right-hand side expression of Eq. (B1) is the integral

$$
\begin{aligned}
\int_{\mathbb{R}} d \mu(z) \int_{\mathbb{R}} d \mu\left(a_{1}\right) \cdots & \int_{\mathbb{R}} d \mu\left(a_{n_{\nu}}\right)\left(\tilde{B}_{1}\right)^{j_{1}}\left(\tilde{M}_{0}\right)^{j_{2}} \\
& \times\left(\tilde{M}_{1}\right)^{j_{3}} \cdots\left(\tilde{M}_{2 m-2}\right)^{j_{2 m}} .
\end{aligned}
$$

Upon close inspection, one notices that the proof presented in Ref. 22 only requires that the measure

$$
d \mu(z) d \mu\left(a_{1}\right) \cdots d \mu\left(a_{n_{\nu}}\right)
$$

be centrally invariant. Other than that, the proof applies to arbitrary distributions of the form

$$
d \mu(z) d \mu_{c i}\left(a_{1}, \ldots, a_{n_{\nu}}\right)
$$

distributions that may not even be products of onedimensional measures. From a short-time approximation of order $\nu$ constructed around Gaussian distributions, one may construct other short-time approximations of order $\nu$ by a change of measure such that

$$
\begin{array}{r}
\int_{\mathbb{R}} d \mu(z) \cdots \int_{\mathbb{R}} d \mu\left(a_{n_{\nu}}\right)\left(\tilde{B}_{1}\right)^{j_{1}}\left(\tilde{M}_{0}\right)^{j_{2}}\left(\tilde{M}_{1}\right)^{j_{3}} \ldots \\
\cdots\left(\tilde{M}_{2 m-2}\right)^{j_{2 m}}=\int_{\mathbb{R}} \ldots \int_{\mathbb{R}} d \mu(z) d \mu_{c i}\left(a_{1}, \ldots, a_{n_{\nu}}\right) \\
\times\left(\tilde{B}_{1}\right)^{j_{1}}\left(\tilde{M}_{0}\right)^{j_{2}}\left(\tilde{M}_{1}\right)^{j_{3}} \ldots\left(\tilde{M}_{2 m-2}\right)^{j_{2 m}},
\end{array}
$$

for all $2 m$-tuples of non-negative integers $\left(j_{1}, j_{2}, \ldots, j_{2 m}\right)$ such that

$$
\sum_{k=1}^{2 m} k j_{k}=2 m \quad \text { and } \quad 1 \leq m \leq \nu .
$$


Observe that the quantities $\tilde{M}_{k}$ are polynomials in the variables $a_{1}, a_{2}, \ldots, a_{n_{\nu}}$ of degree $k$. The integrand in Eq. (B4) is a polynomial of degree

$$
\sum_{k=3}^{2 m}(k-2) j_{k}=2 m-2 \sum_{k=1}^{2 m} j_{k} \leq 2 \nu-2 .
$$

To prove the last inequality, notice that $m \leq \nu$ and that the $j_{k}$ 's cannot be all zero. The maximal degree $2 \nu-2$ is actually attained, for example, for $j_{1}=j_{2}=\cdots=$ $j_{2 \nu-2}=0$ and $j_{2 \nu}=1$. As such, the equalities expressed by Eq. (B4) hold true provided that the measure $d \mu_{c i}\left(a_{1}, \ldots, a_{n_{\nu}}\right)$ integrates exactly all polynomials of degree at most $2 \nu-2$. By central invariance, the polynomials of degree $2 \nu-1$ are also integrated exactly, to zero. The proof of Theorem 2 is concluded.
* Electronic address: cpredescu@comcast.net

1 D. M. Ceperley, Rev. Mod. Phys.67, 279 (1995).

2 R. I. Cukier, Chem. Phys. 305, 197 (2004).

${ }^{3}$ E. Forest and R. D. Ruth, Physica D 43, 105 (1990).

${ }^{4}$ H. Yoshida, Phys. Lett. A 150, 262 (1990).

${ }^{5}$ M. Suzuki, J. Math. Phys. 32, 400 (1991).

6 M. D. Feit, J. J. A. Fleck, and A. Steiger, J. Comput. Phys. 47, 412 (1982).

7 M. D. Feit and J. J. A. Fleck, J. Chem. Phys 78, 301 (1983).

8 R. Kosloff, J. Phys. Chem. 92, 2087 (1988).

9 E. Tadmor, SIAM J. Numer. Anal. 23, 1 (1986).

10 D. Goldman and T. J. Kaper, SIAM J. Numer. Anal. 33, 349 (1996).

11 D. A. Mazziotti, J. Chem. Phys. 115, 6794 (2001).

12 H. Trotter, Proc. Amer. Math. Soc. 10, 545 (1959).

13 R. Cools and P. Rabinowitz, J. Comput. Appl. Math. 48, 309 (1993).

14 R. Cools, J. Comput. Appl. Math. 112, 21 (1999).

15 R. Cools, J. Complexity 19, 445 (2003).

16 N. Victoir, SIAM J. Numer. Anal. 42, 209 (2004).

17 A. D. Klemm and R. G. Storer, Aust. J. Phys. 26, 43 (1973).

18 D. Thirumalai, E. J. Bruskin, and B. J. Berne, J. Chem.
Phys. 79, 5063 (1983).

19 A. Sethia, S. Sanyal and Y. Singh, J. Chem. Phys. 93, 7268 (1990).

20 P. F. Zou, Chem. Phys. Lett. 228, 153 (1994).

21 C. Predescu, http://arxiv.org/abs/cond-mat/0509264.

22 C. Predescu, Phys. Rev. E 69, 056701 (2004).

23 M. Kaluza, Comput. Phys. Commun. 79, 425 (1994).

24 M. Braun, S. A. Sofianos, D. G. Papageorgiou, and I. E. Lagaris, J. Comput. Phys. 126, 315 (1996).

25 J. K. Cullum and R. A. Willoughby, Lanczos algorithms for large symmetric eigenvalue computations, (Birkhauser, Boston, 1985).

${ }^{26}$ B. N. Parlett and D. S. Scott, Math. Comput. 33, 217 (1979).

27 IMSL, Inc., IMSL Math/Library User's Manual, (Houston, Texas).

28 R. Kosloff and H. Tal-Ezer, Chem. Phys. Lett. 127, 223 (1986).

29 S. Flügge, Practical Quantum Mechanics, (SpringerVerlag, New York/Berlin, 1974).

30 T. M. Yamamoto, J. Chem. Phys. 123, 104101 (2005).

31 C. Predescu, Phys. Rev. E 70, 066705 (2004).

32 C. Predescu, Phys. Rev. E 72, 056709 (2005). 